Luigi Alberto Pini

Giuliano Relja

\section{Relevance of analgesic abuse in the maintenance of chronic headaches}

L.A. Pini ( $₫)$

Headache Study Center,

University of Modena and Reggio Emilia,

Via del Pozzo 71, I-41100 Modena, Italy

e-mail:pinila@unimo.it

Tel.: +39-059-4224065

Fax: +39-059-4224069

G. Relja

Neurological Clinic,

Ospedale Maggiore, Trieste, Italy

\begin{abstract}
The mechanisms facilitating or prompting the chronicization of headache and the increased use of analgesics are still unknown and under debate. It is not clear whether the daily use of analgesics in chronic headaches is to be considered a habit or a therapeutic need. Recently, our group showed that items more involved in chronicization of headaches were the onset as migraine and the use of analgesics, namely mixture compounds. One of the most important features in inducing habit behavior is the reward: in this illness, does the reward come from the pain relief or from the intrinsic euphoric activity of the drugs, or does it depend on a more complex behavior? Although the system of neurotransmitters involved in the biology of reward is complex, at least four neurotransmitters are known to be involved at several sites in the brain: serotonin in the hypothalamus, the enkephalins (opioid peptides) in the ventral tegmental area, DOPA in nucleus accumbens, and the inhibitory neurotransmitter GABA in the ventral tegmental area and the nucleus accumbens. In a nor-
\end{abstract}

mal person, these neurotransmitters work together in a cascade of excitation or inhibition between complex stimuli and complex responses leading to a feeling of well being, the ultimate reward. In the cascade theory of reward, a disruption of these intercellular interactions results in anxiety, anger and other "bad feelings" or in a craving for a substance that alleviates these negative emotions. Probably, serotonin plays a pivotal role in migrane pathogenesis, thus drugs alleviating migraine should act through this neurotransmitter. Therefore, these drugs when used in specific settings, as compulsive behavior, may contribute to the development of mechanisms of reward linked to its use and may induce habit behavior. Epidemiological studies support this idea. The chronic use of barbiturate- or opiatecontaining analgesics is preferred by patients who daily consume drugs and who suffer from daily headaches.

Key words Analgesic • Abuse • Reward $\cdot$ Chronic headache

\section{Introduction}

The treatment of headache patients is often complicated by substance abuse that may progress to a drug dependency.
Patients with daily or near-daily headaches often describe a history of daily medication consumption.

The pathogenesis of analgesic-induced headache is not clearly understood, although there is evidence to suggest involvement of serotonin (5HT) in this process [1]. Hering 
et al. [2], in 1993, reported a reduction in whole blood 5HT in patients with analgesic rebound headache which was normalized on discontinuing the analgesics. This rise in 5HT level in the whole blood paralleled the improvement in headache frequency. Patients with analgesic-induced headaches have lower basal content of platelet 5HT; their platelets exhibit reduced 5HT uptake when incubated with excess of the amine, and have a greater density of $5 \mathrm{HT}_{2}-$ receptors on the plasma membrane than migrainous patients without analgesic-reduced headache [1]. These observations suggest that chronic analgesic overuse interferes with the intrinsic pain modulatory system by depletion of 5HT and consequently by up-regulation of its post-synaptic receptors. Therefore, it is likely that analgesic-induced headache is, at least partly, due to defective mechanisms of 5HT uptake caused by analgesic use [3].

The mechanisms facilitating or prompting the chronicization of headache and/or the increased use of analgesics are still unknown and under debate. It is not clear whether the daily use of analgesics is to be considered a habit or simply belongs to therapeutic needs.

Recently, our group showed that items significantly involved in chronicization of headaches were the onset as migraine and the use of analgesics, namely mixture compounds. When we compared the evolution of single patients with respect to analgesic consumption, we identified a strong linkage between the use of mixtures (barbiturate or prochlorperazine-containing drugs) during the follow-up period and the maintenance of this habit along time, whereas only half of patients using single preparations maintained this preference. By contrast about $90 \%$ of patients using mixtures daily did not turn to single compounds.

The constancy in preference for mixtures, both barbiturate and percholperazine-containing compounds, suggests an addictive effect of small doses of these central-acting drugs in maintaining daily drug use.

The International Headache Society (IHS) classifies drugs able to induce abuse and headache. A number of these drugs show antimigraine activity (e.g. NSAIDs, ergotamine) and drug-dependence habit, such as theobromine, caffeine and amphetamine [4].These drugs act by activating the central serotonergic system. Opiates and NSAIDs also act through the serotonergic system, mainly with regard to their antinociceptive activity [5].

Ergot derivatives and triptans act by stimulating $5 \mathrm{HT}$ receptors in the central nervous system (CNS) and are considered to be drugs able to caused drug-induced headaches [6]. The abuse potential of sumatriptan was tested by Sullivan et al. [7] in 1992. This drug was found to be psychoactive, discriminated from placebo, and produced a dose-related decrease in euphoria scores. Elevated scores on measures of apathetic sedation and disliking were found, whereas sumatriptan lacked identification as a prototypic drug of abuse. This study suggested that sumatriptan had only a low abuse potential.

Because the most important feature in inducing habit behavior is the reward, in the case of abuse of analgesics by chronic headache sufferers the main question is: does the reward come from the relief of pain or from the euphoric activity of the drug, or is the behavior more complex? Therefore, reward mechanisms should be an important step in maintaining drug abuse. We outline in this paper the linkage between the serotonergic system in the brain and its potential role in reward mechanisms.

\section{The reward mechanism}

The reward mechanism is a complex reaction to a variety of external and internal stimuli that interact with a number of CNS structures, thereby inducing changes that influence behavior [8]. The pleasure and reward system in the brain was discovered by accident in 1954. The American psychologist James Olds was studying the rat brain's alerting process, when he mistakenly placed the electrodes in a part of the limbic system, a group of structures deep within the brain that are generally believed to play a role in emotions [9]. When the brain was wired so that the animal could stimulate this area by pressing a lever, Olds found that the rats would press the lever almost nonstop, as many as 5000 times per hour. The animals would stimulate themselves to the exclusion of everything else except sleep. They would even endure tremendous pain and hardship for an opportunity to press the lever. Olds had clearly found an area in the limbic system that provided a powerful reward for these animals.

Natural rewards preferentially stimulate dopamine transmission in the nucleus accumbens shell. This effect undergoes adaptive changes (e.g. one-trial habituation, inhibition by appetitive stimuli) that are consistent with a role of nucleus accumbens shell dopamine in associative rewardrelated learning [10]. Experimental studies with a variety of paradigms confirm this role. Resistance to habituation allows drugs to activate dopamine transmission in the shell non-decrementally upon repeated self-administration. It is hypothesized that this process abnormally strengthens stimulus-drug associations, thus resulting in the attribution of excessive motivational value to discrete stimuli or contexts predictive of drug availability. Addiction is therefore the expression of the excessive control over behavior acquired by drug-related stimuli as a result of abnormal associative learning following repeated stimulation of dopamine transmission in the nucleus accumbens shell.

Although the system of neurotransmitters involved in the biology of reward is complex, a number of other neurotransmitters are known to be involved at several sites in the 
brain: serotonin in the hypothalamus, the enkephalins (opioid peptides) in the ventral tegmental area and the nucleus accumbens, and the inhibitory neurotransmitter GABA in the ventral tegmental area and the nucleus accumbens $[8$, 11]. Studies examining the effect of selective agonist and antagonist drugs administered systemically have indicated that multiple neurotransmitters are involved, including dopamine, serotonin, acetylcholine, glutamate, GABA, and various peptides [12]. Direct microinjection studies have also provided crucial evidence indicating that, in addition to the mesolimbic dopamine system, other structures play a role in drug reward, including the ventral pallidum, amygdala, hippocampus, hypothalamus, and pedunculopontine tegmental nucleus. GABAergic circuitry descending from the nucleus accumbens to the pedunculopontine tegmental nucleus via the ventral pallidum appears to be especially important in directing the behavioral sequelae associated with reward produced by various drugs of abuse. However, activation of the reward circuitry is achieved differently for various drugs of abuse.

In a normal person, these neurotransmitters work together in a cascade of excitation or inhibition - between complex stimuli and complex responses - leading to a feeling of well being, the ultimate reward $[11,13]$. In the cascade theory of reward, a disruption of these intercellular interactions results in anxiety, anger and other "bad feelings" or in a craving for a substance that alleviates these negative emotions (Fig. 1).

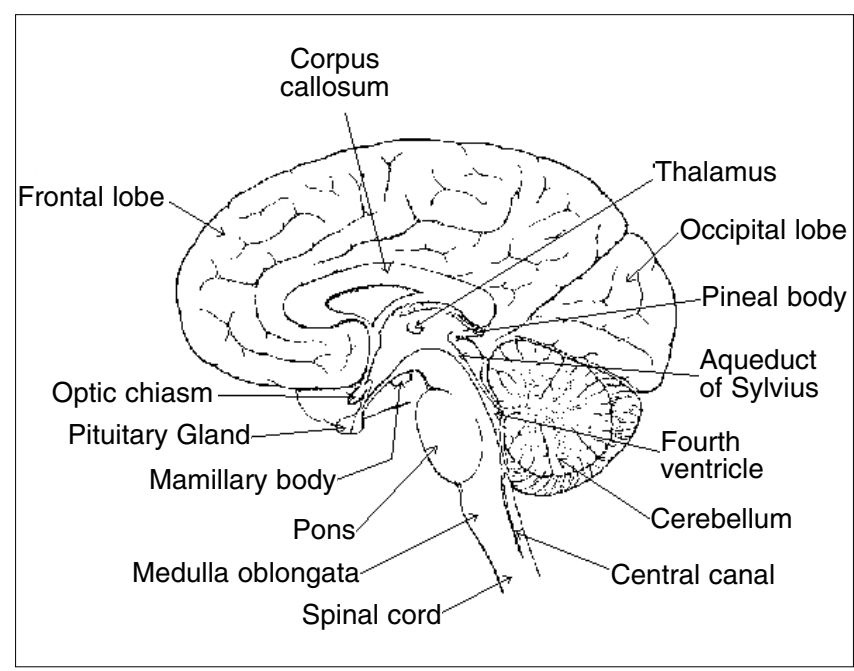

Fig. 1 Structures deep within the limbic system play a crucial role in the expression of emotions and the activity of the reward system of the brain. The experience of pleasure and the modulation of reward are based on a reward "cascade," a chain of neurons within the limbic system that interact through various signaling molecules, or neurotransmitters. We propose that a biochemical deficiency in one or more of these neurons or signaling molecules can supplant an individual's feeling of well being with anxiety, anger or a craving for a substance that can alleviate the negative emotions

\section{Serotonin in the reward system}

$5 \mathrm{HT}_{1}$ recepors play a relevant role in dependence mechanisms [14]. These authors showed that activation of 5-HT $1 \mathrm{~B}$ receptors in the nucleus accumbens reduced amphetamineinduced enhancement of responding for conditioned reward. Moreover, they showed that increasing 5-hydroxytryptamine (5HT) activity attenuated responses for conditioned reward (CR), and that $d$-amphetamine has a response-potentiating effect on this behavior [15]. Serotonin depletion selectively enhances responding for CR. Although 5HT depletion did not potentiate the effects of $d$-amphetamine, it was suggested that CRs activate the mesolimbic dopamine system, and that removal of an inhibitory influence of 5HT on the activity of this system results in increased responding for CR in 5,7-dihydroxytryptamine-treated rats.

Rogers et al. [16] suggested that reduction in central serotonin leads to altered neuromodulation of the cortical and subcortical regions (e.g. orbitofrontal cortex, striatum and anterior temporal structures) that mediate important aspects of associative learning whereby exteroceptive stimuli acquire altered incentive motivational value. On the other hand, facilitation of catecholamine neurotransmitters may disrupt the allocation of attention between relevant and irrelevant features of the environment, perhaps through altered modulation of the dorsolateral prefrontal cortex.

$5 \mathrm{HT}_{3}$ receptors may play a role in reward circuits by modulating the release of dopamine on mesolimbic ways. Mice that overexpress $5 \mathrm{HT}_{3}$ receptors in both cortex and forebrain regions have been developed by placing the gene under the control of the $\mathrm{Ca}^{++}$calmodulin-kinase II $\alpha$ promoter [17]. Overexpression of $5 \mathrm{HT}_{3}$ receptors reduced alcohol consumption in transgenic mice, showing the importance of these receptors in reward behavior. Activation of $5 \mathrm{HT}_{3}$ receptors, the only serotonergic receptor linked to $\mathrm{K}^{+}$and $\mathrm{Na}^{+}$ion channels, changes rapidly the membrane polarization and depletes the neurotransmitter. Antagonists of these receptors reduce mesolimbic dopamine and reduce the psychomotor activity of dopaminergic drugs. In fact, $5 \mathrm{HT}_{3}$ antagonists inhibit withdrawal symptoms of a number of drugs, such as cocaine, amphetamine, morphine and diazepam, but not of alcohol [18]. The experimental evidence suggests that $5 \mathrm{HT}_{3}$ antagonists act through specific neurotransmitter pathways and not through non-specific modifications.

Ondansetron, a classic antagonist of $5 \mathrm{HT}_{3}$ receptors, does not modify behavior induced by nicotine [19].

The role of $5 \mathrm{HT}_{4}$ receptors is not well established, whereas these receptors are located in cerebral areas rich in dopamine receptors. The $5 \mathrm{HT}_{4}$ antagonist SB204070-A did not modify motor behavior in amphetamine- and nicotinetreated rats, thereby excluding a role of this receptor in the motor behavior induced by these drugs [20]. 


\section{Analgesic abuse}

An alteration in serotonin concentration has been found in patients with chronic daily headache $(\mathrm{CDH})$ linked with abuse of analgesic drugs, as has been the up-regulation of $5 \mathrm{HT}_{2}$ platelet receptors, which has been correlated with chronicization of headache [1]. This was correlated with NO and cGMP production and a reduction in platelet serotonin levels. The morphological modifications in platelets and biochemical evaluations lead to the hypothesis that the NO-related intracellular mechanisms operating only in platelets of migraine patients are more accentuated in chronic daily headaches patients owing to chronicization from a previous history of migraine without aura. Whether changes in serotonin and NO metabolism in platelets of $\mathrm{CDH}$ patients reflect similar changes in CNS is under debate. However, it has often considered that platelets can be considered to be a peripheral model of monoaminergic neurons, and a similar alteration of the central serotonergic pathway in $\mathrm{CDH}$ patients cannot be excluded [21].

Nicolodi et al. [22] reported that chronic treatment with 5HT agonists such as methysergide and propranolol ameliorate chronic hadaches; by contrast the use of sumatriptan and ergotamine worsened the clinical picture. These authors concluded by stressing the possible 5HT supersensitivity that characterizes these patients.

In 1996, Schnider et al. [23] in a 5-year follow-up of 38 patients with chronic headache found a close correlation between the frequency of headache and the following: duration of drug abuse, the intensity of headaches and the number of tablets taken per month.

In a 4-year follow-up at the Headache Center of Modena (Italy) (unpublished data), we found that most patients preferred the use of mixtures, both barbiturates and percholperazine-containing compounds. This preference suggests that small doses of central-acting drugs contribute to the reinforcement of drug abuse (Table 1).
Life events indirectly influence the frequency and the intensity of headache, thus modifying behavior and changing the relationships among drug, pain and patient. Finally, a reward emerges from the resolution of pain [24].

In a large study on psychiatric comorbidity in patients with tension-type headache, chronic patients showed high generalized anxiety and psychosocial stress. This emphasizes the need for a systematic investigation of psychiatric comorbidity in these patients [25].

Some patients experience rebound headache from overuse of analgesics and other headache medications. Sumatriptan and dihydroergotamine are more expensive than other migraine agents but have distinct therapeutic advantages in patients with moderate to severe headaches [26].

In 1999, Zed et al. [27] reviewed a number of therapies for acute management of migraine with intervallar headache $(\mathrm{MIH})$, although no rigorously conducted clinical trials were identified. Therapies evaluated included abrupt withdrawal of analgesics, initiation of dihydroergotamine, nonsteroidal antiinflammatory agents, methylergonovine, dihydroergotamine, sumatriptan, amitriptyline, dexamethasone, piracetam, prothipendyl, and valproate. Epidemiology, diagnosis, clinical features, pathophysiology, and longterm prognosis of therapy were discussed and therapeutic guidelines were offered. Serotonin agonists for the treatment of chronic daily headaches have been studied and some positive results have been obtained in follow-ups lasting some months [28, 29].

There is evidence from animals that supports the role of serotonin in nociception. The antinociceptive activity of some NSAIDs has been proved to be mediated by the serotonergic system [5]. The same drugs are also used widely in the treatment of headaches and migraine attacks. Evidence of influence of these drugs on the opiatergic system has also been reported. These data suggest possible links between the serotonergic system and the endogenous opi-

Table 1 Trends in drug abuse in 90 chronic daily headache sufferers through a 4-year follow-up by analgesic type

\begin{tabular}{lrrrrr}
\hline & \multirow{2}{*}{ Abusers at study entry, $\mathrm{n}(\%)$} & \multicolumn{3}{c}{ At 4-year follow-up } \\
\cline { 3 - 6 } & & & Abusers, $\mathrm{n}$ & Non-abusers, $\mathrm{n}(\%)^{\mathrm{a}}$ \\
\hline Optalidon $^{\mathrm{b}}$ & 22 & $(24)$ & 15 & 7 & $(32)$ \\
Difmetrè $^{\mathrm{c}}$ & 17 & $(19)$ & 11 & 6 & $(35)$ \\
Mixtures $^{\mathrm{d}}$ & 7 & $(8)$ & 5 & 2 & $(29)$ \\
NSAIDs & 35 & $(39)$ & 17 & 18 & $(52)$ \\
Triptans & 3 & $(3)$ & 2 & 1 & $(33)$ \\
Ergot & 6 & $(7)$ & 3 & 3 & $(50)$ \\
Total & 90 & $(100)$ & 54 & 36 & $(40)$ \\
\end{tabular}

a Percent of patients that were no longer abusers after 4 years, for the respective drug; ${ }^{b}$ Optalidon: phenazone + caffeine + butalbital; ${ }^{c}$ Dilfmetrè: indomethacin + caffeine + perchlorperazine; ${ }^{d}$ Mixtures: drugs containing paracetamol of aspirin plus caffeine or metilmorphine or metoclopramide 
atergic system, well known to be the main system involved in final modulation of pain control, and on the reward mechanism. In this way the same neurotransmitters play a role in modulating nociception and reward mechanism. This observation suggests that drugs acting on these pathways can induce or favor the reward behavior.

\section{Conclusions}

The main problem with the role of daily analgesic use (or so-called abuse) in the maintenance of chronic headache depends on the lack of animal models in which to evaluate the relationship between drug use and headache. On the other hand clinical well controlled trials are lacking in literature. Objective and ethical reasons do not allow the design of placebo-controlled trials to measure the habit potency of antimigraine drugs in headache sufferers. Moreover, there is some confusion in the terms medication misuse, overuse and abuse-headaches. Mathew [3] in 1998 defined this clinical picture as "a self-sustaining, rhythmic, headache-medication cycle characterized by daily or near daily headache and irresistible and predictable use of immediate relief medications". This sentence contains an ambiguous definition: "the only means of relieving headache attacks" means that the headache attack is on, or should we understand a preventive therapy assumed by the patients for an "imminent" or "forecast probable attack"? In the first case the drug assumption is correct because there is a medical indication to assume analgesic drugs when headache is starting. In the second one the range of variability in the decision to assume or not the drug, to reach an actual preventive therapy of that attack depends in large amount on the emotive status of the patient. In this case it can be considered, when the time and the behavior of the drug assumption are always the same, a compulsive behavior and speculate on a possible abuse.

So the abuse must be considered as a compulsive behavior in some way not strictly linked to the type of used analgesic and related more to "how" the drug is assumed than "what" drug is assumed.
There is no evidence of addictive personality in these patients [30]. Sensation-seeking behavior auditory potentials are lacking in these patients with chronic headache [31]

A number of chronic diseases are associated with prolonged daily or near daily assumpion of analgesic drugs, often belonging to the NSAID pharmacological class. In these patients, for example in patients suffering from chronic arthritis, there were no reports of increased incidence of headache or the existence of habit due to the analgesic use; by contrast analgesic assumption is recommended and the withdrawal of analgesic is evaluated as non-compliance by physicians [32].

In the follow-up performed in our center, the type of abused drugs were constant over time. After 4 years, the percent of drugs that were used daily was not statistically modified, whereas the patients using drugs containing CNS-active compounds maintained the use more than patients assuming simple analgesics.

Reward behavior is a widespread mechanism involving many neurotransmitters and thus is influenced by a large number of drugs, as by a large number of socio-economic conditioning behaviors. In this respect, pharmacologically speaking, drugs used for headache and migraine relief can modify these behaviors. Moreover, the mental significance of head "ache" and the lifestyles related to the presence and the management of migraine and headaches are quite different within patients. So only in migraine sufferers we can identify a clinical picture, so-called analgesic abuse, where the headache disappears when the drugs used to treat the pain are discontinued. In this way we observe a difference between the use of analgesics in patients suffering from chronic arthritis and patients suffering from chronic headaches.

Finally, some reports suggest the chronic sufferers exhibit a compulsive mode of assumption of antimigraine analgesics, i.e. time-scheduled assumption, preventive assumption of analgesic because of a forecast of headache linked to a particular life event (e.g. trip, job, meeting, dinner). This fact may underlie the role of drug significance in abuse or addictive behavior present in chronic headache patients.

\section{References}

1. Srikiatkhachron A, Maneesri S, Govitrapong P, Kasantikul V (1998) Derangement of serotonin system in migraneous patients with analgesic abuse headache: clues from platelets. Headache 38:43-49

2. Hering R, Glover V, Patichis K et al (1993) 5HT in migraine patients with analgesics rebound headache. Cephalalgia 13:410-412
3. Mathew NT (1998) Medication misuse in headadche. Cephalalgia 18[Suppl 21]:21-34

4. Headache Classification Committee of the IHS (1988) Classification and diagnostic criteria for headache disorders, cranial neuralgias and facial pain. Cephalalgia 8:1-96

5. Sandrini M (1999) Central effects of non-opioid analgesics. CNS Drugs $12: 337-345$
6. Silberstein SD, Lipton RB, Goadsby PJ (1998) Headache in clinical practice. Oxford University, Oxford, pp 101-107

7. Sullivan JT, Preston KL, Testa MP, Bush M, Jasinski DR (1992) Psychoactivity and abuse potential of sumatriptan. Clin Pharmacol Ther 52:635-642 
8. Blum K, Cull GC, Braverman, RE, Comings DE (1999) Reward deficiency syndrome. Am Scientist 4:3-8

9. Olds ME, Olds J (1969) Effects of lesions in medical forebrain bundle on self-stimulation behavior. Am J Physiol 217:1253-1264

10. Di Chiara G (1999) Drug addiction as dopamine-dependent associative learning disorder. Eur J Pharmacol 375(1-3):13-30

11. Stein L, Belluzzi J (1986) Second messengers, natural rewards, and drugs of abuse. Clin Neuropharmacol 9[Suppl 4]:205-207

12. Bardo MT (1998) Neuropharmacological mechanisms of drug reward: beyond dopamine in the nucleus accumbens. Crit Rev Neurobiol $12: 1-2$

13. Cloninger CR (1987) A systematic method for clinical description and classification of personality variants. A proposal. Arch Gen Psychiatry 44(6):573-588

14. Fletcher PJ, Korth KM, Chambers JW (1999) Selective destruction of brain serotonin neurons by 5,7-dihydroxytryptamine increases responding for a conditioned reward.

Psychopharmacology Berl 147(3):291-299

15. Fletcher PJ, Korth KM (1999) Activation of 5-HT1B receptors in the nucleus accumbens reduces amphetamine-induced enhancement of responding for conditioned reward. Psychopharmacology 142(2):165-174

16. Rogers RD, Blackshaw AJ, Middleton HC, Matthews K, Hawtin K, Crowley C, Hopwood A, Wallace C, Deakin JF, Sahakian BJ, Robbins TW (1999) Tryptophan depletion impairs stimulusreward learning while methylphenidate disrupts attentional control in healthy young adults: implications for the monoaminergic basis of impulsive behavior. Psychopharmacology Berl 146(4):482-491
17. Engel SR, Lyons CR, Allan AM (1998) 5-HT3 receptor over-expression decreases ethanol self administration in transgenic mice. Psychopharmacology 140:243-248

18. Grant KA (1995) The role of 5-HT3 receptors in drug dependence. Drug Alcohol Dep 38:155-171

19. Ivanova S, Greenshaw AJ (1997) Nicotine-induced decreases in VTA electrical self-stimulation thresholds: blockade by haloperidol and mecamylamine but not scopolamine or ondansetron. Psychopharmacology 134:187-192

20. Reavill C, Hatcher JP, Lewis VA, Sanger GJ, Hagan J (1998) 5-HT4 receptor antagonism does not affect motor and reward mechanisms in the rat. Eur J Pharmacol 357(2-3):115-120

21. Sarchielli P, Alberti A, Russo S, Codini M, Panico R, Floridi A, Gallai V (1999) Nitric oxide pathway, Ca2+ and serotonin content in platelets from patients suffering from chronic daily headache. Cephalalgia 9:S10-S16

22. Nicolodi M, Delbianco PE, Sicuteri F (1997) The way to serotonergic use and abuse in migraine. Int J Clin Pharmacol Res 17:79-84

23. Schnider P, Aull S, Baumgartner C, Marterer A, Wober C, Zeiler K, Wessely P (1996) Long-term outcomes of patients with headache and drug abuse after inpatient withdrawal: fiveyear follow-up. Cephalalgia 16:481-485

24. Fernandez E, Sheffield J (1996) Relative contributions of life events versus daily hassels to the frequency and intensity of headaches. Headache 36:595-602
25. Puca F, Genco S, Prudenzano MP, Savarese M, Bussone G, D'Amico D, Cerbo R, Gala C, Coppola MT, Gallai V, Firenze C, Sarchielli P, Guazzelli M, Guidetti V, Manzoni G, Granella F, Muratorio A, Bonuccelli U, Nuti A, Nappi G, Sandrini G, Verri AP, Sicuteri F, Marabini S (1999) Psychiatric comorbidity and psychosocial stress in patients with tension-type headache from headache centers in Italy. Cephalalgia 19(3):159-164

26. Moore KL, Noble SL (1997) Drug treatment of migraine: Part I. Acute therapy and drug-rebound headache. Am Fam Physician 56(8):2039-2048

27. Zed PJ, Loewen PS, Robinson G (1999) Medication-induced headache: overview and systematic review of therapeutic approaches. Ann Pharmacother 33(1):61-72

28. Diener HC, Dichgans J, Scholtz E, Geiselhart S, Gerber WD, Bille A (1989) Analgesic-induced chronic headache: long-term results of withdrawal thearpy. J Neurol 236:9-14

29. Hering R, Steiner TJ (1991) Abrupt outpatients withdrawal of medication in analgesic-abusing migraneurs. Lancet 337:1442-1443

30. Michultka DM, Blanchard EB, Appelbaum KA, Jaccard J, Detinger MP (1989) The refractory headache patient. II. High medication consumption (analgesic rebound) headache. Behav Res Ther 27:411-420

31. Wang W, Timsit-Berthier M, Schoenen J (1995) Negative correlation between negative seeking behavior and intensity dependence of auditory evoked pontential in migraine. Cephalalgia 15[Suppl 14]:65

32. Bowdler I, Kilian J (1988) The association between analgesic abuse and headache: coincidental or casual. Headache 28:494 